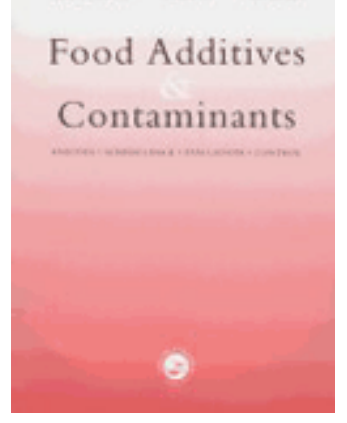

\title{
Validation of a liquid chromatography-tandem mass spectrometry screening method to monitor 58 antibiotics in milk: qualitative approach
}

\begin{tabular}{|r|l|}
\hline Journal: & Food Additives and Contaminants \\
\hline Manuscript ID: & TFAC-2009-120.R1 \\
\hline Manuscript Type: & Original Research Paper \\
\hline Author: & 19-Jun-2009 \\
\hline Complete List of Authors: & $\begin{array}{l}\text { Gaugain-Juhel, Murielle; AFSSA, LERMVD } \\
\text { Delépine, Bernard; AFSSA-LERMVD } \\
\text { Gautier, Sophie; AFSSA-LERMVD } \\
\text { Fourmond, Marie-Pierre; AFSSA-LERMVD } \\
\text { Gaudin, Valerie; AFSSA, LERMVD; AFSSA-LERMVD } \\
\text { Hurtaud-Pessel, Dominique; AFSSA-LERMVD } \\
\text { Verdon, Eric; AFSSA-LERMVD } \\
\text { Sanders, Pascal; AFSSA-LERMVD }\end{array}$ \\
\hline Methods/Techniques: & In-house validation, LC/MS, Method validation, Statistical analysis \\
\hline Additives/Contaminants: & Veterinary drug residues - antibiotics \\
\hline Food Types: & Milk \\
\hline
\end{tabular}

\section{SCHOLARONE ${ }^{m}$ \\ Manuscripts}


1 Validation of a liquid chromatography-tandem mass spectrometry

2 screening method to monitor 58 antibiotics in milk: qualitative

3 approach

4

5

6 M. Gaugain-Juhel ${ }^{\star}$; B. Delépine; S. Gautier ; M.P. Fourmond ; V. Gaudin ;

$7 \quad$ D. Hurtaud-Pessel ; E. Verdon; P.Sanders

8

9

10 Community Reference Laboratory for Antimicrobial Residues, Agence Française de Sécurité

11 Sanitaire des Aliments, Laboratoire d'Etude et de Recherche sur les Médicaments

12 Vétérinaires et les Désinfectants, La Haute-Marche, BP90203, 35302 Fougères Cedex,

13 France

14

15 Corresponding author

16 M. Gaugain-Juhel

$17 \quad T e l: 33(0) 299947878$

18 Email adress : m.gaugain-juhel@fougeres.afssa.fr 


\section{Abstract}

20 A multi-residue method was developed for monitoring antibiotic residues in milk using liquid 21 chromatography coupled to a tandem quadrupole mass spectrometer (LC/MS-MS). Two very 22 short extractions followed by two LC/MS-MS acquisitions allow the screening of 58 antibiotics 23 belonging to 8 different families (penicillins, cephalosporins, sulfonamides, macrolides, 24 lincosamides, aminoglycosides, tetracyclines and quinolones). This method is currently 25 implemented in the laboratory in a qualitative way: i.e. monitoring the presence or absence of 26 residue in a sample and identification of the analyte before the confirmation step. In order to 27 assess the performance of this method, a validation strategy described in an internal 28 guideline for the validation of screening methods was applied. The aim of the validation was 29 to prove sufficient sensitivity of the method to detect all the targeted antibiotics at the level of 30 interest (MRL) at least. According to the EU commission decision 2002/657/EC, the suitable 31 sensitivity of a screening method can be demonstrated when the CC $\beta$ is below or equal to 32 the MRL level and so the false compliant rate below or equal to $5 \%$ at the MRL level. The 33 validation scheme was established in order to take into account various variability factors: the 34 apparatus response, the interday repeatability, the matrix effect, etc ... The results of the 35 validation clearly demonstrate the suitability of this method for the detection and identification 36 of more than 50 antibiotics and are in agreement with the results obtained in routine analysis.

39 Keywords: antibiotic residues; multi-residue screening; LC/MS-MS; Validation 


\section{Introduction}

The frequent but regulated use of antimicrobial drugs for veterinary purposes may leave residues in edible tissues which can lead to allergic reaction, disorder of intestinal flora or emergence of resistant strains of bacteria. Antibiotics can be used for therapeutic purposes or as feed additives to promote animal growth. In the EU, food safety regulations were established during the 90's (EC 1990, EC 2002) and European Member States are requested to monitor the presence of antimicrobial residues in different tissues of animal origin by implementing suitable residue control programmes.

Microbiological inhibitory plate test methods are mostly used for the screening of antibiotic residues. The advantages of these methods are the possibility to detect a wide number of compounds in a simple way and at a low cost. But these methods are in some cases not enough sensitive (false negative results) and not really specific (false positive results). Furthermore, even if these screening methods are sometimes able to give an indication on the antibiotic family, a further post-screening step is needed and often implemented in order to determine the identity of the previously detected inhibitory substance. Rapid test kits are also employed but have the drawback to cover only a few targeted compounds or compounds belonging to the same family of antibiotics.

LC/MS-MS techniques are now widely used for screening purposes and these methods are able to cover a high number of veterinary drugs (Gergov et al. 2003, Granelli et al. 2007, Hammel et al. 2008, Stolker et al. 2007, Muñoz et al. 2005, Turnipseed et al. 2008). If the high number of transitions to be monitored was previously requiring fragmentation of the acquisition into several retention time windows (segments), the level of sensitivity of the new generation of LC/MS-MS instruments allows performing the acquisition in a single segment. More recently in the mid of the 2000's, a trend appeared in using accurate mass full scan MS techniques (e.g. LC-ToF-MS, LC-LTQ-Orbitrap-MS...) allowing the screening of predefined 
67 compounds but also the research and identification of untargeted compounds (Stolker et al. 2008, Kaufmann et al. 2007, Nielen et al. 2007).

70 This work describes a simple and rapid LC/MS-MS post-screening method which was 71 developed first in muscle tissues and then applied to milk and is currently tested in real-life 72 conditions following a microbiological inhibitory screening step. This method is dedicated to 73 the detection and identification of 58 antibiotics using LC/MS-MS with an electrospray 74 ionisation (ESI) source set in a positive mode and was validated first in muscle tissues and 75 then in raw milk. The screened antibacterials belong to the following families: beta-lactam 76 (penicillins and cephalosporins), tetracyclines, macrolides, quinolones, sulfonamides, 77 lincosamides and aminoglycosides.

78

Although primarily dedicated to a qualitative and identificative screening of authorized antimicrobial substances, this method can be upgraded to a confirmatory step with quantitative results for certain families by adding suitable internal standards in order to decrease and correct matrix effects. This particular issue will be published in other articles from the same authors. Sticking to a screening/post-screening purpose, it was so decided not to use the classical validation approach designed for a quantitative method (accuracy, repeatability and reproducibility...) but to implement a design suitable for the validation of a qualitative method as described in an internal guideline for the validation of screening methods (Gaudin V. 2007). The LC/MS-MS qualitative method as well as the validation scheme and the results of the validation are comprehensively presented in this paper.

\section{Materials and methods}

\section{Chemical, reagents and solutions}

Antibiotic standards were for most of them obtained from Sigma-Aldrich (France) and used for the preparation of individual stock standard solutions (concentration of $1 \mathrm{mg} \cdot \mathrm{ml}^{-1}$ ) in either 
94 methanol or water depending on the solubility of each antibiotic. Solutions of $1-5 \mu \mathrm{g} \cdot \mathrm{ml}^{-1}$

100 All the water used was ultra-pure deionised water produced by a Milli-Q apparatus (Millipore, 101 France). Trichloroacetic acid (TCA) from Fisher Scientific (France), ammonium acetate from were prepared in the mobile phase from the stock solutions for the tuning of the ESI source and for MS/MS transitions settings. From these stock solutions, suitable concentrations of spiking solutions were also prepared in ultra-pure water to be used during the validation process. Merck (France) and pentafluoropropionic acid (PFPA) from Acros Organics (France) were analytical reagent grade. Acetonitrile obtained from Fisher Scientific (France) was a HPLC grade solvent. A 5\% TCA solution was obtained by dissolving $50 \mathrm{~g}$ of trichloroacetic acid in 1 $\mathrm{L}$ of water. A $2 \mathrm{M}$ ammonium acetate solution was prepared by dissolving $15.4 \mathrm{~g}$ of ammonium acetate in $100 \mathrm{~mL}$ of water. This solution was then diluted by tenth to obtain a 0.2 M solution. A $0.1 \%$ PFPA solution was also prepared by adding $1 \mathrm{~mL}$ of pentafluoropropionic acid in $900 \mathrm{~mL}$ of water and by adjusting to $1 \mathrm{~L}$ with water.

\section{Sample preparation}

Two routes of extraction followed by two acquisition modes allow the screening and differentiating of all 58 antibiotics. Acetonitrile was the extracting solvent chosen for the extraction of penicillins, cephalosporins, macrolides and sulfonamides and the $5 \%$ TCA solution was the acidic aqueous solvent chosen for the extraction of tetracyclines, aminoglycosides, lincosamides and quinolones. Sulfaphenazole served as internal standard in both extractions. Raw milk was allowed to thaw followed by a quick homogenisation of the fat by shaking before taking up a test portion of $2 \mathrm{ml}$ to which $200 \mu \mathrm{l}$ of internal standard and $800 \mu \mathrm{l}$ of water were added.

\section{Extraction of penicillins, cephalosporins, macrolides and sulfonamides}


120 After addition of $8 \mathrm{ml}$ of acetonitrile, the samples were stirred for 10 minutes and then

121 centrifuged at $14000 \mathrm{~g}$ for $5 \mathrm{~min}$. After evaporation of $6 \mathrm{ml}$ of the supernatant under nitrogen

122 flow, the residual volume was dissolved in $0.6 \mathrm{ml}$ of $0.2 \mathrm{M}$ ammonium acetate, then filtered 123 onto a $0.45 \mu \mathrm{m}$ Millex HV filter of $13 \mathrm{~mm}$ diameter (Millipore, France). A volume of $25 \mu \mathrm{l}$ were 124 injected and analysed by the LC/MS-MS instrument.

125 Extraction of tetracyclines, quinolones, aminoglycosides and lincomycin

126 After addition of $8 \mathrm{ml}$ of $5 \%$ TCA solution, the sample was stirred for $10 \mathrm{~min}$ and then 127 centrifuged at $14000 \mathrm{~g}$ for 5 minutes. About $1 \mathrm{ml}$ of the supernatant was filtered onto a 0.45 $128 \mu \mathrm{m}$ Millex HV filter. A volume of $20 \mu \mathrm{l}$ was injected and analysed by the LC/MS-MS 129 instrument.

\section{$131 \quad$ LC/MS-MS analysis}

132 An HPLC system (Agilent-HP1100) was used for the chromatographic separation which was 133 performed on a Symmetry ${ }^{\circledR} \mathrm{C} 18$ column, $5 \mu \mathrm{m}, 150 \times 3.9 \mathrm{~mm}$ (Waters) with a security guard 134 system C18, $5 \mu \mathrm{m}, 4.0 \times 3.0 \mathrm{~mm}$ (Phenomenex). Two different gradients mixing $0.1 \%$ PFPA 135 (A) with acetonitrile (B) were used according to the appropriate series of antibiotics. The 136 gradient optimized for the separation of penicillins, cephalosporins, macrolides and 137 sulfonamides started with $10 \%$ of $\mathrm{B}$. It was then rising linearly to $30 \%$ of $\mathrm{B}$ over 4 min, then 138 stoped for $1 \mathrm{~min}$ at $30 \%$ and again rising linearly to $70 \%$ of B over 2 min and stoped for $3 \mathrm{~min}$ 139 at $70 \%$. The initial composition was then recovered over a 1 min delay. The gradient elution 140 for the separation of tetracyclines, quinolones, aminoglycosides and lincomycin started with $14110 \%$ of $\mathrm{B}$, then rising to $50 \%$ of $\mathrm{B}$ over $7 \mathrm{~min}$ and stoped for $4 \mathrm{~min}$ at $50 \%$. The initial 142 composition was then recovered over a 1 min delay.

144 A triple quadripole mass detector (AB-Sciex-API4000) set in a positive ESI mode was used 145 for the detection and identification of the targeted antibiotics. The Turbolon Spray Voltage 146 was set at $5.5 \mathrm{kV}$ and the temperature of the source at $700^{\circ} \mathrm{C}$. MRM conditions, declustering 
147 potential (DP) and collision energy (CE) were first optimized for each antibiotic by infusing 148 solutions of the antibiotic standards prepared in the mobile phase. MRM conditions are

149 displayed in Table la and Table Ib. The presence of two MRM transitions with a signal to 150 noise ratio $(\mathrm{S} / \mathrm{N})$ above 3 in combination with the expected retention time guarantee the 151 univocal identification of the analyte. Even if only one transition can satisfactorily be

152 considered as sufficient for screening purposes, it is preferred to keep monitoring two 153 transitions in such a way to facilitate the technical evaluation of the signals and the analysis 154 of the resulting data through the "Analyst" software onto the instrument. Moreover, watching 155 at two transitions at the same retention time on the computer screen is really more efficient 156 especially when monitoring a large number of samples at screening step.

\section{Validation scheme}

160 The validation process was established according to a guideline set for the comprehensive

161 validation of biological screening methods (Gaudin et al. 2007). This scheme of validation in

162 line with the criteria of the Decision No 2002/657/EC which is more dedicated to quantitative 163 confirmatory methods, was applied to an LC/MS-MS method used as a qualitative post164 screening tool. It was considered more suitable than the classical approach of validation 165 usually applied to quantitative physicochemical methods (trueness, precision, linearity...). In 166 fact, there was no interest in checking the accuracy of the method in terms of quantification.

167 It was considered more relevant to monitor many samples at the level of interest in order to 168 assess statistically the capacity of detection of the method. Anyway, the aim of a validation is 169 to prove the suitability of the method in achieving the goal it is developed for. The only 170 requirement for screening methods as described in the Commission Decision No $1712002 / 657 / E C$ is that these methods have the capability for a high sample throughput and 172 shall allow the detection of all targeted substances with a false compliant rate below $5 \%$ at 173 the level of interest. It means that the $\mathrm{CC} \beta$ of the method should be found below this level of 
174 interest. The way to assess the detection capability is described below as well as the way the

175 limit of detection, the specificity and the sensitivity are calculated respectively. In the case of

176 a suspected non-compliant result, this result shall be confirmed by a confirmatory method.

177

178 Determination of " $T$ " value

179 A first step in assessing the capacity of detection is the calculation of the "T" value for each

180 compound of interest. The "T" value is a "Threshold" value corresponding to the minimum

181 analytical response above which the sample will be truly considered as positive. In order to

182 determine this "T" value, 20 blanks of raw milks from different origins were tested with the

183 method described above. The analytical blank response at the retention time corresponding

184 to the analyte $( \pm 10 \%)$ was determined in each blank chromatogram for the two MRM

185 transitions. The mean value of the noise "B" was then calculated. The threshold value "T"

186 was estimated for each transition as being three times the mean value obtained from the 20

187 recorded noises (see figure 1).

188

189 Determination of the limit of detection:

190 The limit of detection is the smaller concentration of analyte above which it is possible to 191 detect this analyte with a reasonable statistical certainty. By comparing the response 192 corresponding to the "T" value ( 3 times the mean noise) with the mean response of two 193 samples spiked at a known concentration, it s possible to determine the "limit of detection" of 194 the method for each analyte. As the presence of the two transitions is recommended for full 195 identification of the substance when possible, the limit of detection is generally estimated 196 from the results of the most variable response from the two transitions. Very often, this is the 197 minor transition that gives also the most variable one.

198

199 Determination of the cut-off factor "Fm" and assessment of the detection capability (CC beta) 
200 The 20 blanks of raw milk were spiked at the level of interest (MRL or $1 / 2 M R L$ ) with the 201 different analytes and tested within the same day. Generally, the samples were spiked at the 202 MRL level. The half-MRL level was chosen for some compounds especially when the $M R L$ is 203 established for the parent drug plus its metabolite or for the sum of different compounds (e.g. 204 sulfonamides). Stock solutions for each antibiotic were prepared in methanol except for 205 aminoglycosides and penicillins which were dissolved in water. Spiking solutions were 206 prepared in water by mixing the different standards from one antibiotic family. Then, 207 validation was carried out per family in order to get information about the potential 208 interferences. The concentrations evaluated during the validation are given in Table Ila and 209 Table Ilb.

[insert table Ila and IIb about here]

211 This step was repeated again twice in order to get a total of 60 independent data for each 212 analyte at the level of interest. The repetitions were carried out on three different days 213 distributed over three different weeks in order to increase the factors of variability and to 214 mimic routine analysis. The analytical response $(\mathrm{Yi}=$ height of the peak response) were 215 determined for each of the samples $(n=60)$ and for the two MRM transitions from each 216 analytes. Both the mean response from the 60 samples and the corresponding standard 217 deviation were calculated. A "cut-off factor" Fm was then estimated as being the mean 218 response decreased by 1.64 times the standard deviation. It statistically means that $95 \%$ of 219 the samples spiked at the level of interest should give an analytical response above this 220 value (see figure 1).

[insert figure 1 about here]

223 The "T" and "Fm" values obtained can lead to different situations:

$224 \mathbf{F m}>\mathbf{T}$ : this is the best situation corresponding to a false negative rate below $5 \%$. The 225 consequence is the $C C \beta$ is truly below the MRL level. 
$226 \mathbf{F m}<\mathrm{T}$ : if the "T" value is taken as a limit of positivity, then more than $5 \%$ of the samples will 227 be considered as negative. The consequence is the $\operatorname{CC} \beta$ is truly above the MRL level. 228 Further studies at concentrations above the MRL should be implemented in order to 229 determine the gap between the MRL and the CC $\beta$.

234 requirement expected from a screening method is to avoid as much as possible the false 235 negative results also called "false compliant" results.

Assessment of the specificity and sensitivity:

238 The following definitions are needed to understand the calculation of the two parameters 239 "specificity" and "sensitivity". At first, a true positive sample giving a positive test result is 240 called a "positive agreement". Then a true negative sample giving a negative test result is 241 called a "negative agreement". A true positive sample giving a negative test result is called a 242 "false negative" sample. Finally, a true negative sample giving a positive test result is called a 243 "false positive" sample.

245 The "specificity" of a method is the ability of this method to distinguish one specific analyte 246 from the other analytes. It can be determined as being the number of negative agreement 247 divided by the number of true negative samples expressed as a percentage. The "sensitivity" 248 of a method can be determined as being the number of positive agreements divided by the 249 number of true positive samples expressed as a percentage. A sample is considered to be 250 positive as soon as the signal ranges above the "T" value (three times the mean noise). A 251 sensitivity above $95 \%$ means that the $\operatorname{CC} \beta$ is below the level of concentration tested for the 252 validation. Then the number of false negatives is truly below $5 \%$. 


\section{Results and discussion}

255 Sample pretreatment and LC/MS-MS analysis

256 The challenge for the analyst in the development of a multi-residue method was to find 257 suitable extraction conditions for a large range of targeted analytes displaying different 258 chemical properties (lipophilicity, hydrophilicity, alcaline and acidic characteristics, ...). As it 259 is almost impossible to optimize the cleanup process for all analytes, the purification step has 260 generally to be as short and simple as possible. Having a look at the literature in the field of 261 veterinary drug residues, two main routes of extraction were considered in muscle. The first 262 one was acetonitrile extraction which is a common solvent used for many veterinary drugs 263 including antibiotics. This solvent was found to be sufficiently effective for the extraction of 264 macrolides, sulfonamides, penicillins, and cephalosporins. The second route was an acidic 265 extraction with trichloroacetic acid which was found to be suitable for quinolones, 266 aminoglycosides, lincomycin, and tetracyclines. Results obtained when comparing the 267 different solvents evaluated for extraction of the 58 antimicrobials were not always in full 268 agreement with the expected results taking into consideration the chemical properties of the 269 different analytes. But as previously mentioned, the aim of this study was to find global 270 conditions acceptable for all substances and not to optimize the recovery for each analyte. 271 These extraction conditions were then tested successfully on milk. The difficulty to find a 272 generic extraction solvent is due to the aminoglycoside family for which acidic extractions are 273 mainly used. During the validation, spiked samples were prepared with twenty different 274 batches of milk in order to check suppression or enhancement effects due to the matrix. A 275 variability due to matrix effects was sometimes observed, particularly for the hydrophilic 276 compounds eluting in the first minutes after injection, but validation results proved that the 277 sensitivity was always sufficient to detect all the analytes at the level of interest. PFPA and 278 acetonitrile in a gradient mode were chosen as mobile phase for the chromatographic 279 separation. Even if it is announced that PFPA may lead to ion suppression effects in 
280 electrospray ionization and if the ionization rate was not optimized for all analytes with this 281 acidic solvent, this last was preferably selected for its advantageous ion-pairing properties 282 which allowed including in this method as strong bases as the aminoglycosides and as 283 relatively strong acids as amoxicillin. In contradiction with the general trend to 284 chromatography with narrow-bore analytical columns, a conventional Symmetry ${ }^{\circledR}$ analytical 285 column (Waters, France) of $3.9 \mathrm{~mm}$ in diameter was chosen. It was considered in this study 286 that the use of a narrower column with a lower flow-rate did not preclude saving time even if 287 it effectively saves solvent. In fact, due to the sloping gradient used, the period for stabilizing 288 the column between to injections was found time-consuming. Satisfactorily, using the 289 Symmetry ${ }^{\circledR}$ analytical column with a $3.9 \mathrm{~mm}$ diameter, no stabilization time was needed 290 between injections and the runs were finally shorter ( $<15$ minutes). The high selectivity and 291 sensitivity of the triple-quadruple detector allowed the detection of 58 analytes in two runs 292 without segmenting the acquisition. The singly-charged precursor ion was preferentially 293 considered for all the 58 antimicrobial analytes and the two highest MRM transitions were 294 then monitored for each analyte. Some sulfonamides displaying isobaric masses and giving 295 the same ionic fragments were nevertheless identified taking into account their different 296 retention times. Some chromatograms are presented in figure2.

[Insert figure 2 about here]

298 Method validation

299 The validation process previously described had already been tested for biological screening 300 methods but was totally new for a LC/MS-MS method. The suitability of this validation 301 scheme applied to a physicochemical screening method was demonstrated and gave more 302 useful information than the classical approach of validation usually applied to quantitative 303 physicochemical methods. The limit of detection, the specificity, sensitivity and the CCbeta 304 were assessed during the validation and the results are described below for each antibiotic 305 family. The specificity of the method was checked by analyzing twenty milk blank samples of 306 different origins and the specificity was of $100 \%$ for all the analytes as no peak was detected 
307 in these samples at the retention time corresponding to each analyte. This fact underlines the 308 very high specificity of the tandem mass spectrometer.

Tetracyclines

311 Milk samples were spiked at $50 \mu \mathrm{g} \cdot \mathrm{kg}^{-1}$ for oxytetracycline, 4-epi-oxytetracycline, tetracycline,

312 4-epi-tetracycline, chlortetracycline and 4-epi-chlortetracycline as the MRL is $100 \mu \mathrm{gg}^{-\mathrm{kg}^{-1}}$ for 313 the sum of the parent drug and its epimer. The concentration of validation for doxycycline 314 was $100 \mu \mathrm{g} \cdot \mathrm{kg}^{-1}$. Twenty spiked samples were analysed per day and during three days. In 315 total, 60 samples were analysed for each analyte. Results are presented in Table III and are 316 very satisfactory as the "Fm" values are above the "T" values for all analytes. These results 317 mean that the CCbeta of the 7 tetracyclines are below the level of concentrations tested 318 during the validation: i.e. below $1 / 2 \mathrm{MRL}$ for OTC, TTC, and CTC and their 4-epimers and 319 below MRL for DC. The limits of detection were calculated and are ranging between 0.3 and $3202.2 \mu \mathrm{g} \cdot \mathrm{kg}^{-1}$ for the first transition and between 1.5 and $13.6 \mu \mathrm{g} \cdot \mathrm{kg}^{-1}$ (4-epi-tetracycline) for the 321 second "minor" transition. The sensitivity was calculated at $100 \%$ for all the tetracyclines and 322 their epimers. Retention times were ranging from 7.2 to $8.1 \mathrm{~min}$.

[insert table III about here]

\section{Sulfonamides and macrolides}

326 The level of validation for each analyte is given in Table 2a. According to the validation 327 scheme, 60 spiked samples were analysed on three different days. The estimated CCbeta 328 was below the level of validation for all sulfonamides and macrolides as the "Fm" value were 329 above the "T" values. The limits of detection were below $1 \mu \mathrm{g} \cdot \mathrm{kg}^{-1}$ for most of the analytes 330 and up to $1.7 \mu \mathrm{g} \cdot \mathrm{kg}^{-1}$ for sulfaguanidine (minor transition). The sensitivity was $100 \%$ for all 331 sulfonamides and macrolides. Retention times were ranging from 3.1 to $9.6 \mathrm{~min}$ for 332 sulfonamides and from 7.3 to $9.8 \mathrm{~min}$ for macrolides. 


\section{Quinolones}

335 The use of norfloxacin and nalidixic acid is not allowed in veterinary medicine for food336 producing animals but a concentration of validation of $100 \mu \mathrm{g} \cdot \mathrm{kg}^{-1}$ was arbitrarily chosen. The 337 MRL for the sum of enrofloxacin and its metabolite ciprofloxacin being $100 \mu \mathrm{g} \cdot \mathrm{kg}^{-1}$, a 338 concentration of validation of $50 \mu \mathrm{g} \cdot \mathrm{kg}^{-1}$ was chosen for each. The level of validation for other 339 quinolones is given in Table 2b. Results were very satisfactory for this family as the

340 estimated CCbeta was below the level of validation for all quinolones except for oxolinic acid 341 and the sensitivity of $100 \%$ for all (for the two transitions). It means that oxolinic acid was 342 always detected at the level of validation but there was enough variation in the response to 343 assess a low "Fm" value. The limits of detection of the quinolones were below $1 \mu \mathrm{g} \cdot \mathrm{kg}^{-1}$ and 344 up to $1.95 \mu \mathrm{g} \cdot \mathrm{kg}^{-1}$ for the major transition and below $1 \mu \mathrm{g} \cdot \mathrm{kg}^{-1}$ and up to $15.5 \mu \mathrm{g} \cdot \mathrm{kg}^{-1}$ for the 345 minor transition (sarafloxacin). Retention times were ranging from 7.0 to $11.1 \mathrm{~min}$.

$347 \quad$ Aminosides

348 The levels of validation for each aminoglycoside are given in Table $2 \mathrm{~b}$. Results were very 349 satisfactory as the CC $\beta$ was assessed to be below the level of validation for all analytes 350 except for spectinomycin (minor transition). The limits of detection were calculated and 351 ranged between 0.2 and $3.5 \mu \mathrm{g} \cdot \mathrm{kg}^{-1}$ for the first transition and between 0.2 and $66 \mu \mathrm{g} \cdot \mathrm{kg}^{-1}$ 352 (spectinomycin) for the minor transition. The sensitivity was 100\% for all aminosides except 353 for the minor transition of spectinomycin. Retention times were ranging from 5 to $6.5 \mathrm{~min}$.

355 Penicillins and cephalosporins

356 The concentrations of validation for these two families are given in Table $2 \mathrm{a}$ and results for 357 penicillins are presented in Table VI. Results were very satisfactory for penicillins and 358 cephalosporins except for amoxicillin for which the $C C \beta$ was assessed to be above the MRL 359 of $4 \mu \mathrm{g} \cdot \mathrm{kg}^{-1}$ for the three chosen transitions. Nevertheless, the sensitivity was calculated as 360 being above $95 \%$ for the second and third transition and we can so ensure a sufficient 
361 detection capability by considering that the presence of at least one transition is enough to 362 consider a further confirmatory step. In the same way, the $\operatorname{CC} \beta$ was above the MRL for the 363 minor transition of ampicillin. The $C C \beta$ obtained for the cephalosporins were all below the 364 concentration of validation except for the first transition of cefoperazone but the sensitivity 365 was $100 \%$ for all of them. Retention time were ranging from 6.9 to 11.6 min for penicillins and 366 from 6.9 to $9.4 \mathrm{~min}$ for cephalosporins.

[insert table VI about here]

\section{Validation results}

370 The results of the validation clearly demonstrate the suitability of this method for the 371 detection and identification of all tested antibiotics. Particular attention should be paid to the 372 following compounds for which the detection is often problematic: i.e. amoxicillin, ampicillin, 373 and spectinomycin. However, for these compounds, the presence of at least one transition 374 shall lead to the confirmatory step. For amoxicillin, a careful observation of the 375 chromatograms at the retention time of interest shall be carried out.

377 Participation to different proficiency tests organized in the field of antibiotic residues in order 378 to demonstrate the efficiency of this method, clearly showed a very satisfactory evaluation of 379 its capacity of detecting and identifying antibacterial residues as no false-negative and no 380 false-positive results were obtained.

\section{Field of application}

383 Application of this method to milk was presented in this paper but the method was first 384 developed for muscle tissue samples. Used in routine analysis as a post-screening method 385 carried out after a first microbiological screening step, it allows the full identification of the 386 antibiotic previously responsible of the microbiological activity inhibition. The step for 387 quantitative confirmation is then more efficient and not so time-consuming. On the side of 
388 analytical quality assurance, it was not easily conceivable to implement quality control 389 samples spiked at the level of interest for the 58 antibiotics in such a routine use, particularly 390 because of stability problems with penicillin solutions. Therefore, after the validation step, our 391 choice in order to assure a sufficient sensitivity in routine analysis was to add an internal 392 standard for each sample to be analyzed and to set a minimal height of signal response to 393 qualify the analysis according to the validation data. Furthermore, a sample matrix spiked 394 with one analyte from each family was added as quality control sample. The criteria for the 395 quality control have to be set in relation to the results obtained from the validation step. Then 396 the follow-up of a control chart provides a reliable checking of the suitability for these criteria. 397 The high correlation between the microbiological screening results and the mass 398 spectrometry post-screening results demonstrates the high efficiency of this method in 399 routine use, especially here described for milk. Nevertheless, a few samples being detected 400 positive at the microbiological step were not confirmed to be positive at the post-screening 401 step. There are two possibilities : the microbiological result could be a false positive result or 402 the concerned analyte was not monitored by the LC/MS-MS method. One of the 403 disadvantages of LC-triple quadripole MS instruments compared to LC-ToF-MS instruments 404 is the need to optimize for each new compound before being included in the acquisition 405 mode of the method. Moreover, there is no possibility to review and reassess the analytical 406 data collected by the instrument on a previously analyzed sample when it is necessary for 407 exemple to check for the presence of one suspected "untargerted" analyte if this analyte was 408 not initially included in the method.

410 This multi-residue method is not suitable for confirmatory purposes in the here-described 411 format of the method as quantitative requirements from the Decision No 2002/657/EC are not 412 always met for some particular families. For example, large matrix effects in muscle tissue 413 were observed for tetracycline residues. Quantification of macrolides was also not accurate 414 enough with the here-above described extraction. Some dilutions of the samples and use of 
415 suitable internal standards could improve the accuracy of the method within the context of a 416 quantitative confirmation. For penicillins, cephalosporins and quinolones, minor changes and 417 the use of internal standard(s) may allow using this method as a confirmatory method. 


\section{Conclusion}

419 The LC/MS-MS method proposed in this article allows a rapid and efficient screening or post-

420 screening of 58 antibiotics in milk and meets the requirements of EU/2002/657 for screening 421 methods as demonstrated during the validation. One possibility to increase further the 422 sensitivity of the method will be to implement a new acquisition mode as it is proposed in the 423 new version of the LC/MS-MS software Analyst version 1.5 which is the so-called "MRM 424 scheduled" mode. This mode allows the automatic implementation of retention time windows 425 which is useful for the detection of a high number of analytes. The sensitivity is enhanced by 426 setting longer acquisition dwell-times for each compound. It is planned to extend the field of 427 application of this LC/MS-MS multi-antibiotic method by including the monitoring to other 428 antibiotics used in veterinary medicine but not yet included in the method as bacitracin, 429 colistin, trimethoprim. It was proved during this study that LC-triple quadripole MS instrument 430 offers suitable opportunities to implement a reliable monitoring in the well-defined regulatory 431 context of food safety in the EU.

\section{References}

434 EC 1990. Council Regulation (EEC) N²377/90 of 26 June 1990: laying down a Community 435 procedure for the establishment of maximum residue limits of veterinary medicinal products 436 in foodstuffs of animal origin. Official Journal of European Communities L224:1-8.

437 EC 2002. Commission Decision 2002/657/EC of 12 August 2002: implementing Council 438 Directive 96/23/EC concerning the performance of analytical methods and the 439 interpretation of results. Official Journal of European Communities L221:8-36.

440 Gergov M, Ojanperä I, Vuori E. 2003. Simultaneous screening for 238 drugs in blood by 441 liquid chromatography-ionspray tandem mass spectrometry with multiple reaction 442 monitoring. Journal of chromatography B $795: 41-53$. 
443 Granelli K, Branzell C. 2007. Rapid multi-residue screening of antibiotics in muscle and 444 kidney by liquid chromatography-electrospray ionization-tandem mass spectrometry. $445 \quad$ Analytica Chimica Acta 586: 289-295.

446 Hammel YA, Mohamed R, Gremaud E, Lebreton MH, Guy PA. 2008. Multi-screening 447 approach to monitor and quantify 42 antibiotics residues in honey by liquid 448 chromatography-tandem mass spectrometry. Journal of Chromatography A 1177: 58$449 \quad 76$.

450 Stolker AAM, Zuidema T, Nielen MWF. 2007. Residue analysis of veterinary drugs and 451 growth-promoting agents. Trends in Analytical Chemistry 26: 967-979.

452 Muñoz P, Blanca J, Ramos M, Bartolomé M, Garcia E, Méndez N, Gomez J, Martin de 453 Pozuelo M. 2005. A versatile liquid chromatography-tandem mass spectrometry 454 system for the analysis of different groups of veterinary drugs. Analytica Chimica Acta $455 \quad 529: 137-144$.

456 Turnipseed SB, Andersen WC, Karbiwnyk CM, Madson MR, Miller KE. 2008. Multi-class, 457 multi-residue liquid chromatography/tandem mass spectrometry screening and 458 confirmation method for drug residues in milk. Rapid Communications in Mass $459 \quad$ Spectrometry $22: 1467.1480$.

460 Stolker AAM, Rutgers P, Oosterink E, Lasaroms JJP, Peters RJB, Van Rhijn JA, Nielen $461 \quad$ MWF. 2008. Comprehensive screening and quantification of veterinary drugs in milk 462 using UPLC-ToF-MS. Analytical and Bioanalytical Chemistry 391: 2309-2322.

463 Kaufmann A, Butcher P, Maden K, Widmer M. 2007. Ultra-performance liquid 464 chromatography coupled to time of flight mass spectrometry (UPLC-TOF): A novel tool 465 for multiresidue screening of veterinary drugs in urine. Analytica Chimica Acta 586: 1346621.

467 Nielen MWF, Van Engelen MC, Zuiderent R, Ramaker R. 2007. Screening and confirmation 468 criteria for hormone residue analysis using liquid chromatography accurate mass time- 
469 of-flight, Fourrier transform ion cyclotron resonance and orbitrap mass spectrometry

$470 \quad$ techniques. Analytica Chimica Acta 586: 122-129.

471 Gaudin V, Sanders P. 2007. Guide pour la validation des méthodes biologiques de dépistage 472 version 1, AFSSA-LERMVD, internal document, unpublished. 
Figure 1. Graphical representation of threshold value "t" and Cut-off factor "Fm"

Figure 2. Examples of chromatograms of milk samples spiked at the EU-MRL or half the EU-MRL levels 
Figure 1

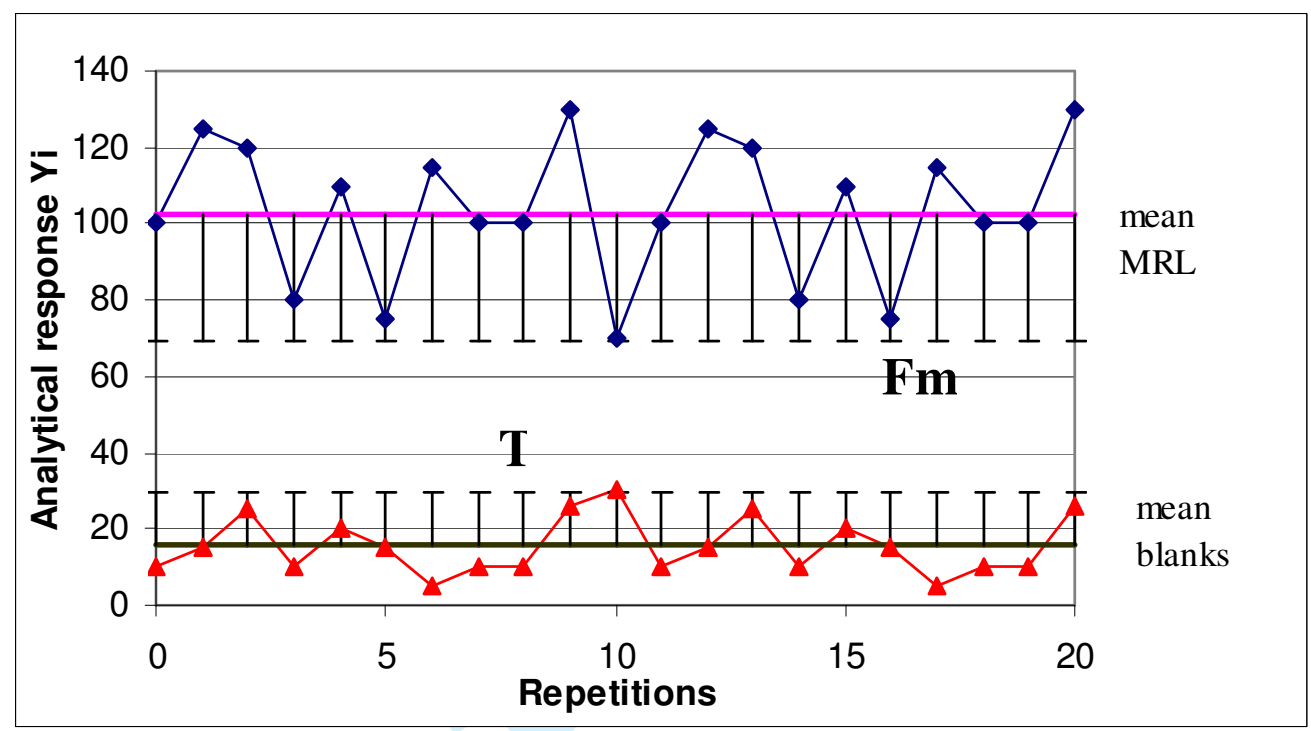


Figure 2

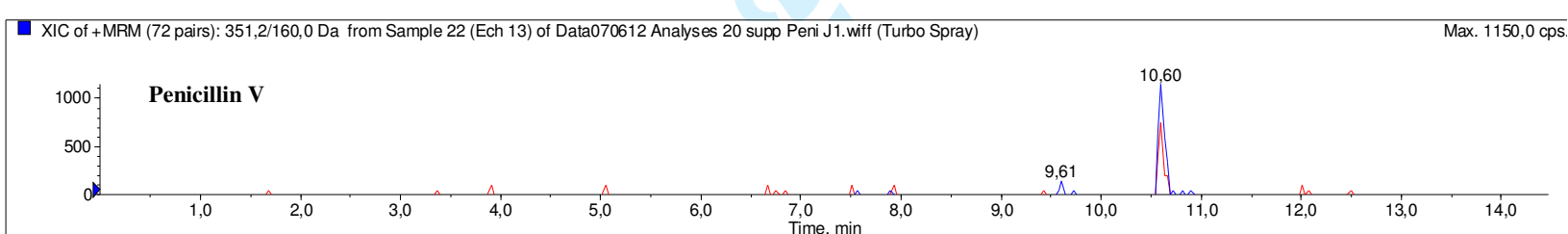

XIC of +MRM (72 pairs): 335,1/160,0 Da from Sample 22 (Ech 13) of Data070612 Analyses 20 supp Peni J1. wiff (Turbo Spray)

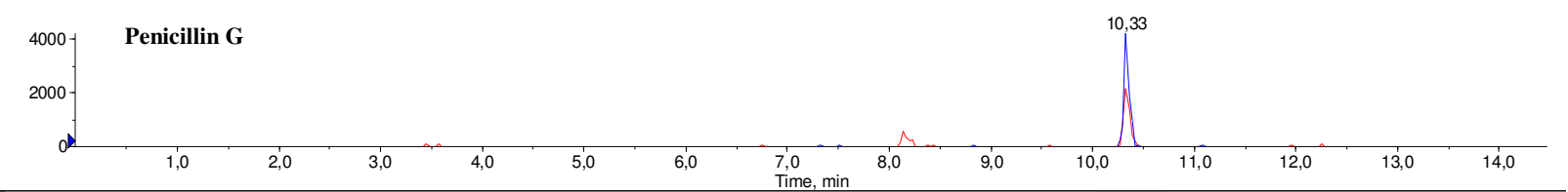

XIC of +MRM (72 pairs): 350,3/106,0 Da from Sample 22 (Ech 13) of Data070612 Analyses 20 supp Peni J1.wiff (Turbo Spray)

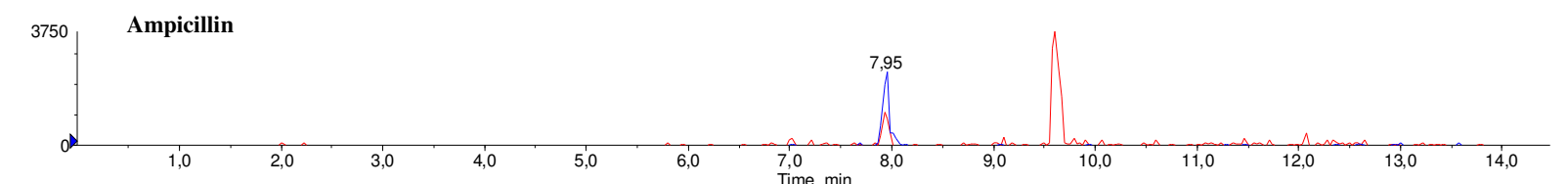

XIC of +MRM (72 pairs): 366,1/114,0 Da from Sample 22 (Ech 13) of Data070612 Analyses 20 supp Peni J1.wiff (Turbo Spray) Max. 450,0 cps

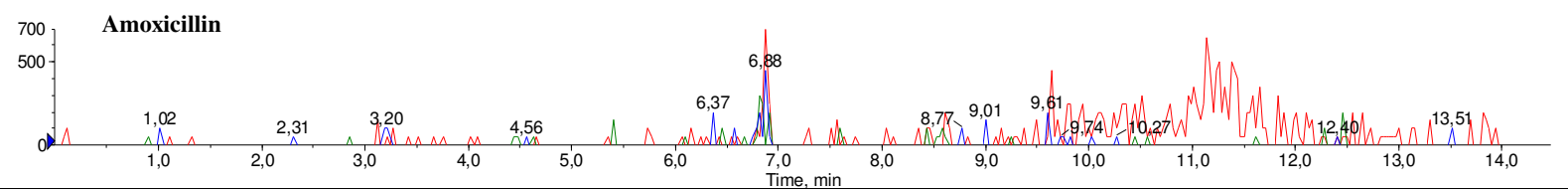




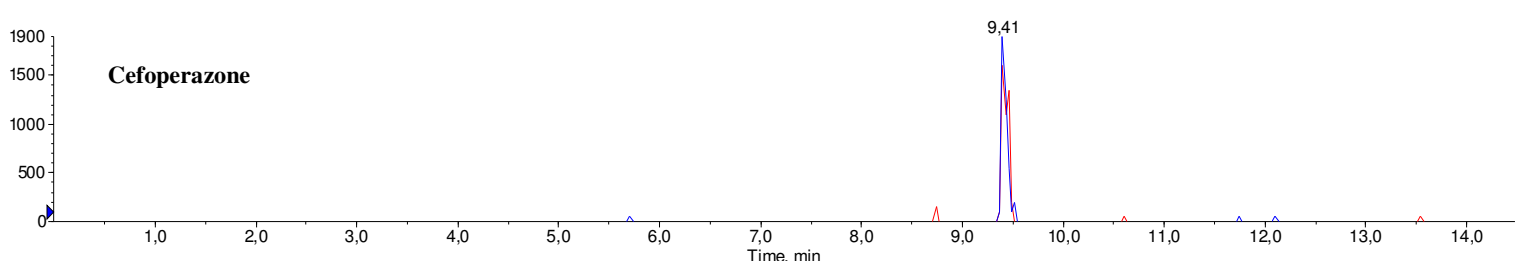

- XIC of +MRM (72 pairs): 348,0/158,0 Da from Sample 17 (Ech 10) of Data070724 Analyses 20 sup Cephalo J3.wiff (Turbo Spray)

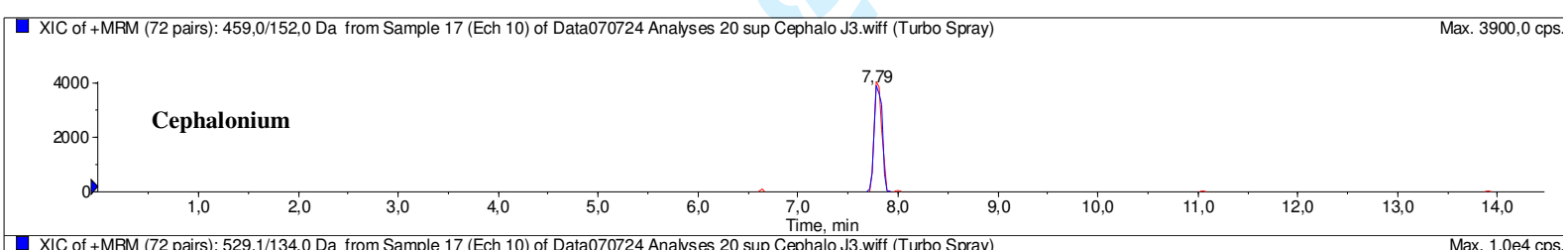

XIC of +MRM (72 pairs): 529,1/134,0 Da from Sample 17 (Ech 10) of Data070724 Analyses 20 sup Cephalo J3.wiff (Turbo Spray)

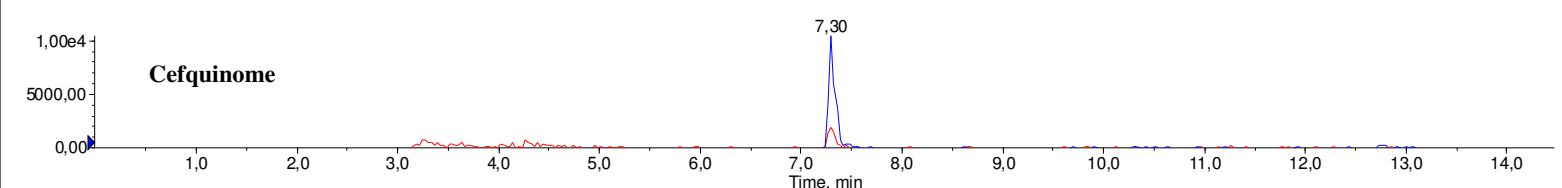

- XIC of +MRM (72 pairs): 424,0/152,0 Da from Sample 17 (Ech 10) of Data070724 Analyses 20 sup Cephalo J3.wiff (Turbo Spray)

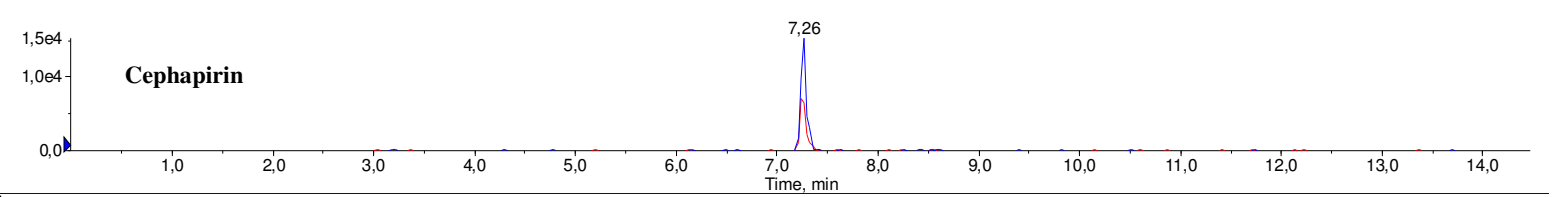

- XIC of +MRM (72 pairs): 548,9/183,1 Da from Sample 17 (Ech 10) of Data070724 Analyses 20 sup Cephalo J3.wiff (Turbo Spray)

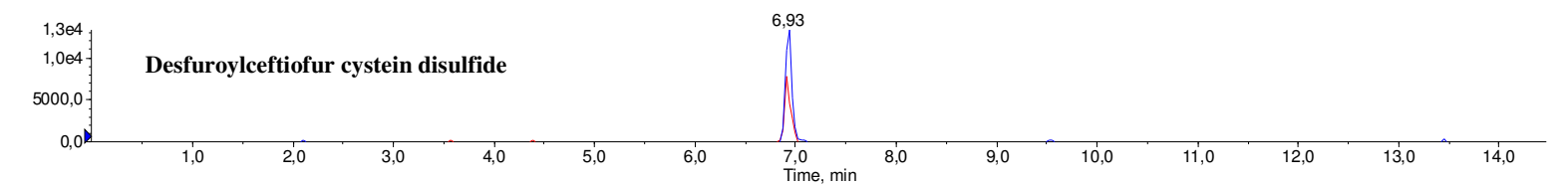




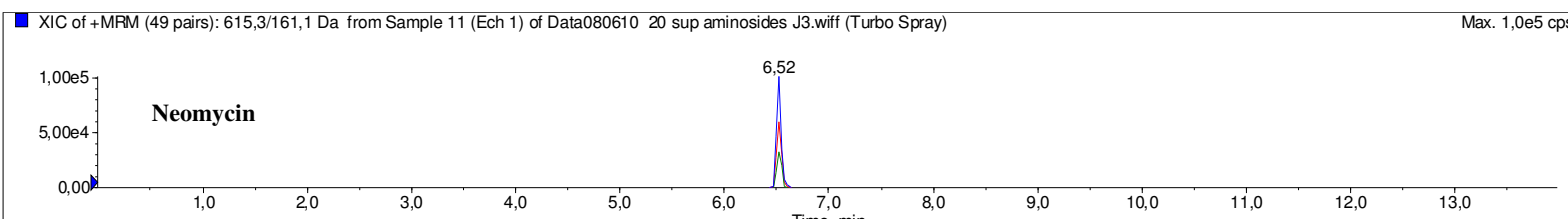

XIC of +MRM (49 pairs): 450,3/322,0 Da from Sample 11 (Ech 1) of Data080610 20 sup aminosides J3.wiff (Turbo Spray)

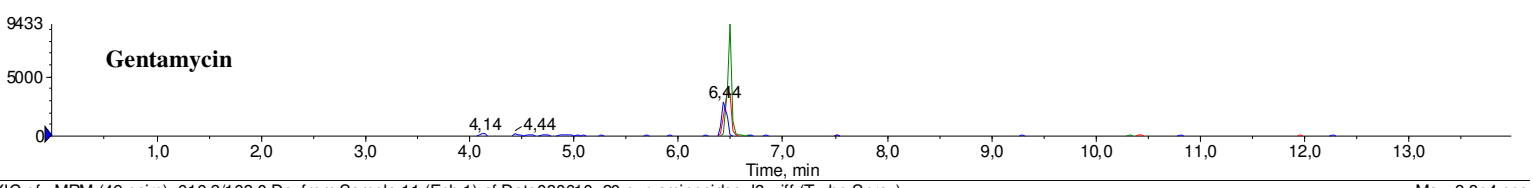

XIC of +MRM (49 pairs): 616,3/163,0 Da from Sample 11 (Ech 1) of Data080610 20 sup aminosides J3.wiff (Turbo Spray)

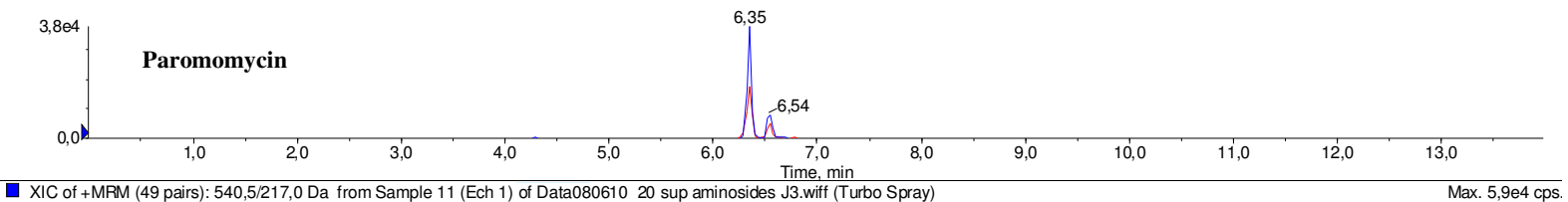

- XIC of +MRM (49 pairs): 540,5/217,0 Da from Sample 11 (Ech 1) of Data080610 20 sup aminosides J3.wiff (Turbo Spray) Max. 5,9e4 cps.
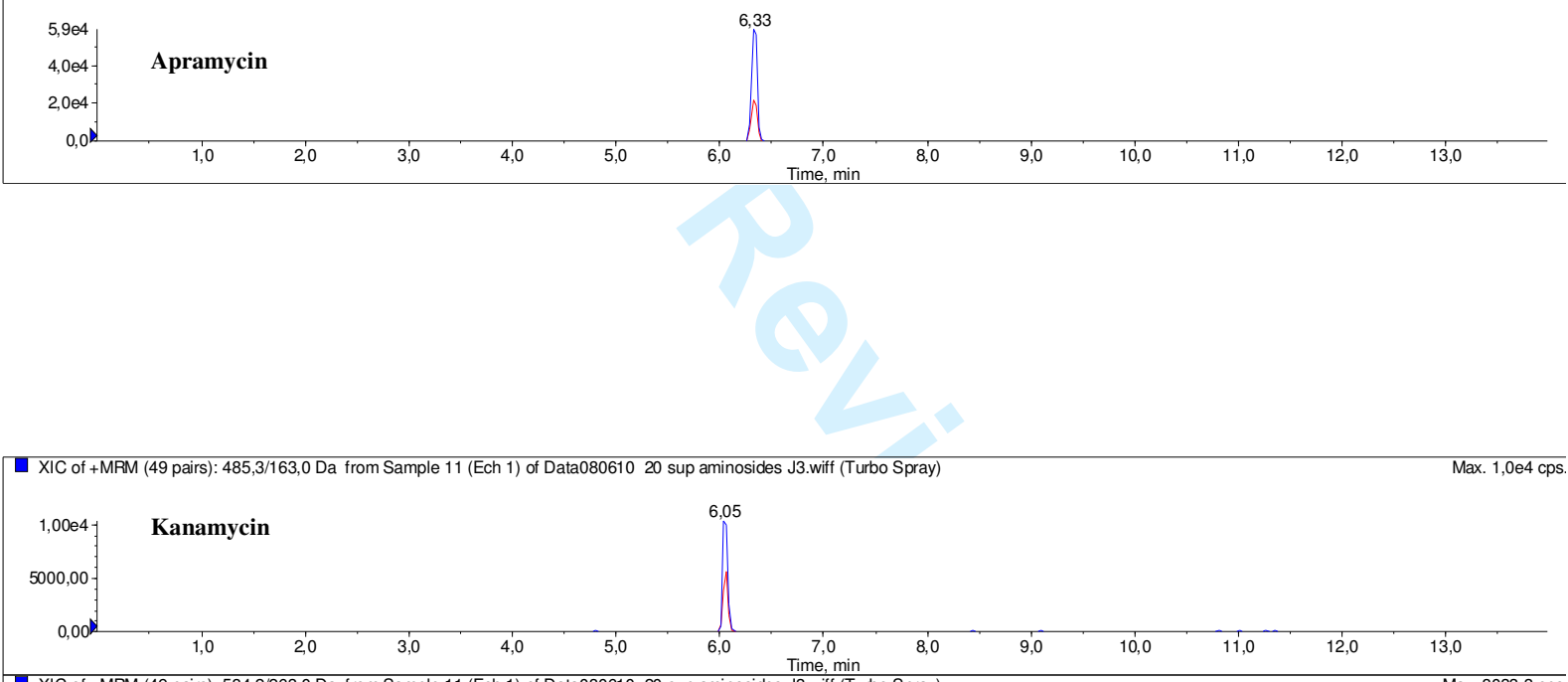

- XIC of +MRM (49 pairs): 584,2/263,0 Da from Sample 11 (Ech 1) of Data080610 20 sup aminosides J3.wiff (Turbo Spray)

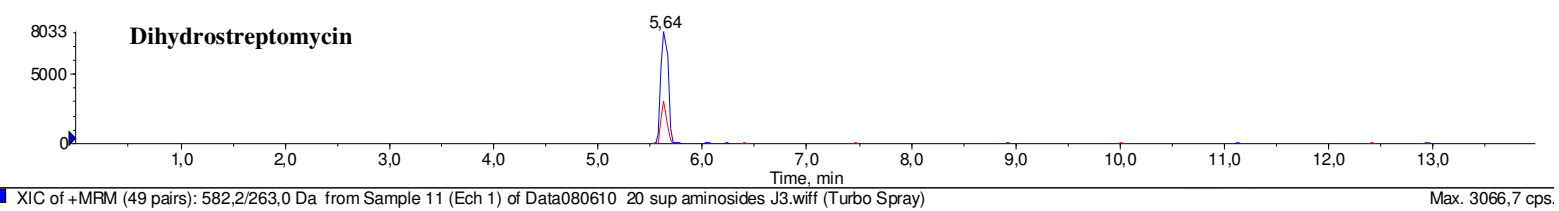

XIC of +MRM (49 pairs): 582,2/263,0 Da from Sample 11 (Ech 1) of Data080610 20 sup aminosides J3.wiff (Turbo Spray)

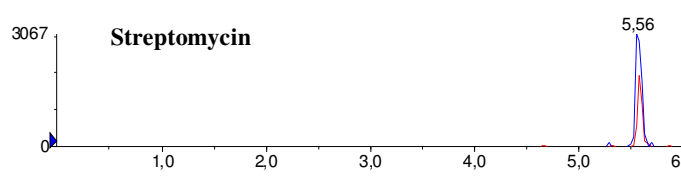

- XIC of +MRM (49 pairs): 351,4/333,0 Da from Sample 11 (Ech 1) of Data080610 20 sup aminosides J3.wiff (Turbo Spray)

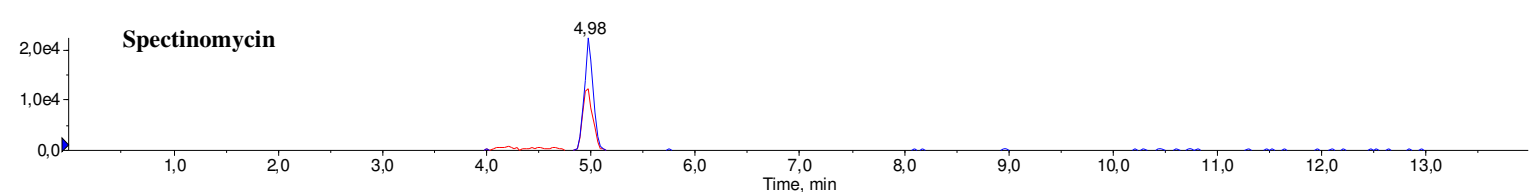


Table Ia. MRM transitions for cephalosporins, penicillins, sulfonamides and macrolides in the acetonitrile extract

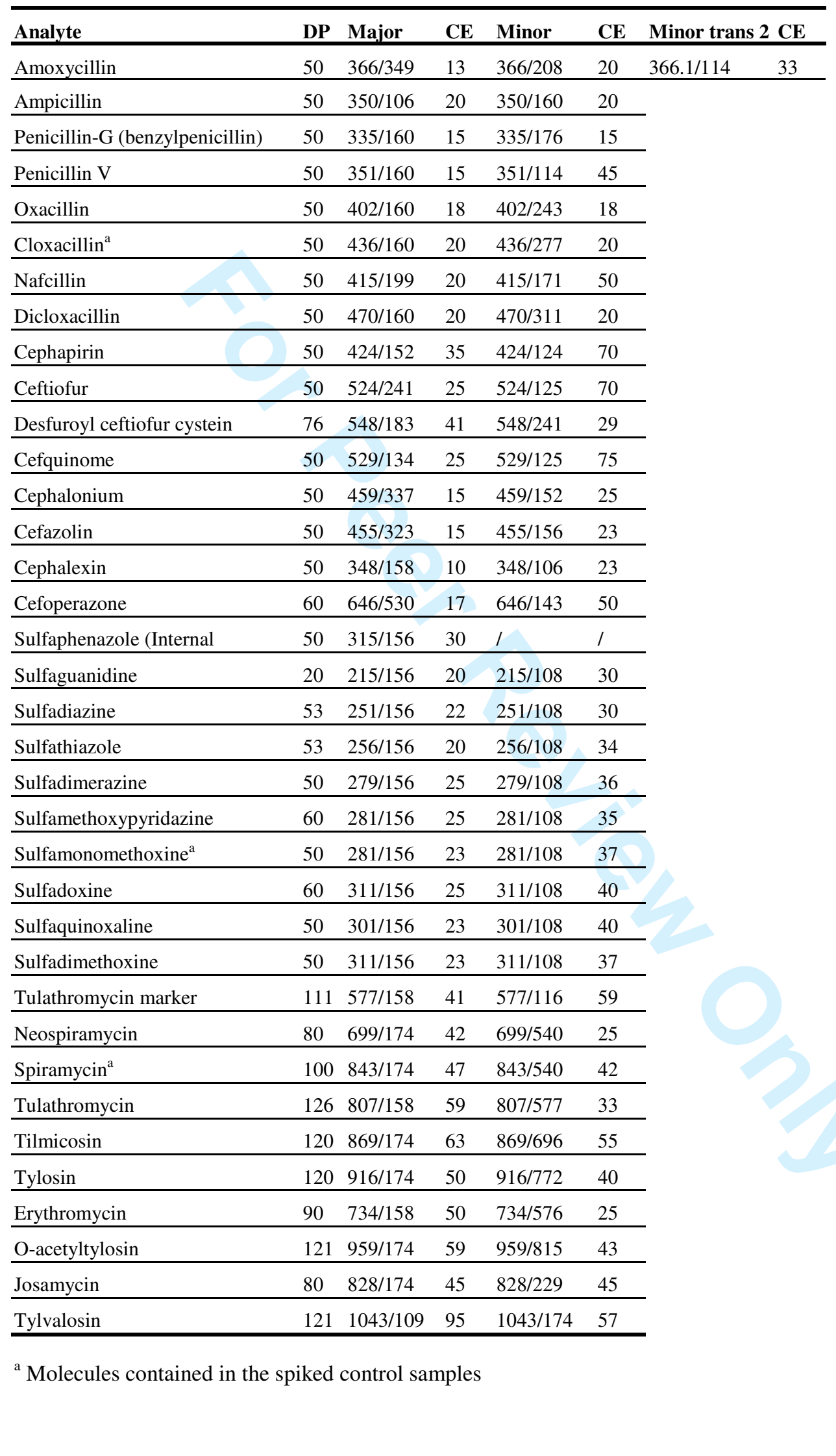


Table Ib. MRM transitions for quinolones, aminoglycosides, lincosamides and tetracyclines in the trichloroacetic acid extract

\begin{tabular}{|c|c|c|c|c|c|}
\hline Analyte & DP & Major Trans & CE & MinorTrans & $\mathbf{C E}$ \\
\hline Spectinomycin & 71 & $351 / 333$ & 27 & $351 / 207$ & 31 \\
\hline Streptomycin & 157 & $582 / 263$ & 45 & $582 / 246$ & 51 \\
\hline Dihydrostreptomycin $^{\mathrm{a}}$ & 120 & $584 / 263$ & 42 & $584 / 246$ & 54 \\
\hline Kanamycin & 70 & $485 / 163$ & 35 & $485 / 205$ & 35 \\
\hline Paromomycin & 84 & $616 / 163$ & 45 & $616 / / 293$ & 33 \\
\hline Gentamycin $\mathrm{C} 1, \mathrm{C} 1 \mathrm{a}$ et $\mathrm{C} 2$ & 60 & $478 / 322,450 / 322,464 / 322$ & 20 & & \\
\hline Neomycin & 92 & $615 / 161$ & 45 & $615 / 163$ & 30 \\
\hline Apramycin & 82 & $540 / 217$ & 35 & $540 / 378$ & 25 \\
\hline Lincomycin & 60 & $407 / 126$ & 40 & $407 / 359$ & 26 \\
\hline Oxytetracycline $^{\mathrm{a}}$ & 65 & $461 / 426$ & 30 & $461 / 443$ & 17 \\
\hline Tetracycline & 55 & $445 / 410$ & 27 & $445 / 427$ & 25 \\
\hline Chlortetracycline & 60 & $479 / 444$ & 29 & $479 / 462$ & 23 \\
\hline Doxycycline & 55 & $445 / 428$ & 25 & $445 / 154$ & 40 \\
\hline Marbofloxacin & 70 & $363 / 345$ & 30 & $363 / 320$ & 22 \\
\hline Norfloxacin & 60 & $320 / 302$ & 33 & $320 / 231$ & 50 \\
\hline Ciprofloxacin $^{a}$ & 61 & $332 / 314$ & 30 & $332 / 231$ & 47 \\
\hline Danofloxacin & 60 & $358 / 340$ & 33 & $358 / 255$ & 50 \\
\hline Enrofloxacin & 72 & $360 / 342$ & 30 & $360 / 286$ & 50 \\
\hline Sarafloxacin & 50 & $386 / 368$ & 30 & $386 / 348$ & 50 \\
\hline Difloxacin & 80 & $400 / 382$ & 30 & $400 / 356$ & 50 \\
\hline Oxolinic acid & 53 & $262 / 244$ & 25 & $262 / 216$ & 40 \\
\hline Nalidixic acid & 42 & $233 / 215$ & 30 & $233 / 187$ & 35 \\
\hline Flumequine & 44 & $262 / 244$ & 25 & $262 / 202$ & 45 \\
\hline Sulfaphenazole (Standard Interne) & 50 & $315 / 156$ & 30 & & \\
\hline
\end{tabular}

${ }^{\mathrm{a}}$ Molecules contained in the spiked control samples 
Table IIa. EU-MRL and level of concentration for the validation of the analytes extracted by acetonitrile

\begin{tabular}{|c|c|c|}
\hline Analyte & MRL in milk $(\mu \mathrm{g} / \mathrm{kg})$ & $\begin{array}{c}\text { Validation concentration (Cval) } \\
(\mu \mathrm{g} / \mathrm{kg})\end{array}$ \\
\hline Sulfaguanidine & 100 & 50 \\
\hline Sulfadiazine & 100 & 50 \\
\hline Sulfathiazole & 100 & 50 \\
\hline Sulfadimerazine & 100 & 50 \\
\hline Sulfamethoxypyridazine & 100 & 50 \\
\hline Sulfamonomethoxine & 100 & 50 \\
\hline Sulfadoxine & 100 & 50 \\
\hline Sulfaquinoxaline & 100 & 50 \\
\hline Sulfadimethoxine & 100 & 50 \\
\hline Tulathromycin marqueur & Banned $^{\mathrm{a}}$ & 25 \\
\hline Neospiramycin & $200^{\mathrm{b}}$ & 100 \\
\hline Spiramycin & $200^{\mathrm{b}}$ & 100 \\
\hline Tulathromycin & Banned $^{\mathrm{a}}$ & 25 \\
\hline Tilmicosin & 50 & 50 \\
\hline Tylosin & $\theta^{2}$ & 50 \\
\hline Erythromycin & 40 & 40 \\
\hline O-Acétyltylosin & $1^{\mathrm{c}}$ & 25 \\
\hline Josamycin & $/^{\mathrm{c}}$ & 25 \\
\hline Tylvalosin & $/^{\mathrm{c}}$ & 25 \\
\hline Amoxicillin & 4 & 4 \\
\hline Ampicillin & 4 & 4 \\
\hline Penicillin G (benzylpenicillin) & 4 & 4 \\
\hline Penicillin V (phenoxymethylpenicillin) & l & 4 \\
\hline Oxacillin & 30 & 30 \\
\hline Cloxacillin & 30 & 30 \\
\hline Nafcillin & 30 & 30 \\
\hline Dicloxacillin & 30 & 30 \\
\hline Cefquinome & 20 & 20 \\
\hline Cefalonium & 20 & 20 \\
\hline Cephapirin + desacetylcephapirin & 60 & 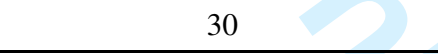 \\
\hline Cefoperazone & 50 & 50 \\
\hline Cefazolin & 50 & 50 \\
\hline Desfuroylceftiofur cystein disulfide & 100 & 100 \\
\hline Cephalexin & 100 & 100 \\
\hline
\end{tabular}

${ }^{\mathrm{a}}$ Banned for use in milk producing-animals

${ }^{\mathrm{b}}$ MRL established for the parent drug + the metabolite

${ }^{\mathrm{c}}$ No MRL in milk 
Table IIb. EU-MRL and level of concentration for the validation of the analytes extracted by trichloroacetic acid

\begin{tabular}{|c|c|c|}
\hline Analyte & MRL in milk ( $\mu \mathrm{g} / \mathrm{kg})$ & $\begin{array}{c}\text { Validation concentration (Cval) } \\
(\mu \mathrm{g} / \mathrm{kg})\end{array}$ \\
\hline Spectinomycin & 200 & 200 \\
\hline Streptomycin & 200 & 200 \\
\hline Dihydrostreptomycin & 200 & 200 \\
\hline Kanamycin & 150 & 150 \\
\hline Paromomycin & Banned $^{\mathrm{a}}$ & 500 \\
\hline Gentamycine $\mathrm{C} 1, \mathrm{C} 1 \mathrm{a}$ and $\mathrm{C} 2$ & 100 & 100 \\
\hline Neomycin & 1500 & 1500 \\
\hline Apramycin & $/^{\mathrm{c}}$ & 1000 \\
\hline Lincomycin & 150 & 150 \\
\hline Oxytetracycline (+ 4-epimer) & 100 & 50 \\
\hline Tetracycline (+ 4-epimer) & 100 & 50 \\
\hline Chlortetracycline (+ 4-epimer) & 100 & 50 \\
\hline Doxycycline & $1^{\mathrm{c}}$ & 100 \\
\hline Marbofloxacin & 75 & 75 \\
\hline Norfloxacin & $l^{\mathrm{d}}$ & 100 \\
\hline Danofloxacin & 30 & 30 \\
\hline Ciprofloxacin & $100^{\mathrm{b}}$ & 50 \\
\hline Enrofloxacin & $100^{\mathrm{b}}$ & 50 \\
\hline Sarafloxacin & $/^{\mathrm{c}}$ & 30 \\
\hline Difloxacin & Banned $^{\mathrm{a}}$ & 300 \\
\hline Oxolinic acid & Banned $^{\mathrm{a}}$ & 100 \\
\hline Nalidixic acid & $I^{\mathrm{d}}$ & 100 \\
\hline Flumequine & 50 & 50 \\
\hline \multicolumn{3}{|c|}{${ }^{a}$ Banned for use in milk producing-animals } \\
\hline \multicolumn{3}{|c|}{${ }^{\mathrm{b}}$ MRL established for the parent drug + the metabolite } \\
\hline${ }^{\mathrm{c}}$ No MRL in milk & & \\
\hline${ }^{\mathrm{d}}$ No authorisation in veterina & nedicine & \\
\hline
\end{tabular}


Table III. Results of the validation for tetracyclines

\begin{tabular}{|c|c|c|c|c|c|c|c|c|}
\hline \multirow[b]{2}{*}{ Analyte } & \multicolumn{4}{|c|}{ Transition 1 « major » } & \multicolumn{4}{|c|}{ Transition 2 « minor » } \\
\hline & $\mathbf{F m} / \mathrm{T} / \mathrm{B}$ & CCbeta & $\operatorname{LOD}(\mu \mathrm{g} / \mathrm{kg})$ & Sensitivity (\%) & $\mathbf{F m} / \mathbf{T} / \mathbf{B}$ & CCbeta & LOD $(\mu \mathrm{g} / \mathrm{kg})$ & Sensitivity (\%) \\
\hline Oxytetracycline & $\mathrm{Fm}>\mathrm{T}$ & $<$ MRL & $<1$ & $100 \%$ & $\mathrm{Fm}>\mathrm{T}$ & $<$ MRL & 2.98 & $100 \%$ \\
\hline Tetracycline & $\mathrm{Fm}>\mathrm{T}$ & $<\mathrm{MRL}$ & 1.41 & $100 \%$ & $\mathrm{Fm}>\mathrm{T}$ & $<$ MRL & 4.68 & $100 \%$ \\
\hline Chlortetracycline & $\mathrm{Fm}>\mathrm{T}$ & $<$ MRL & $<1$ & $100 \%$ & $\mathrm{Fm}>\mathrm{T}$ & $<\mathrm{MRL}$ & 1.47 & $100 \%$ \\
\hline Doxycycline & $\mathrm{Fm}>\mathrm{T}$ & $<$ MRL & 3.33 & $100 \%$ & $\mathrm{Fm}>\mathrm{T}$ & $<\mathrm{MRL}$ & 3.04 & $100 \%$ \\
\hline 4-epi-oxytetracycline & $\mathrm{Fm}>\mathrm{T}$ & $<$ MRL & $<1$ & $100 \%$ & $\mathrm{Fm}>\mathrm{T}$ & $<\mathrm{MRL}$ & 1.67 & $100 \%$ \\
\hline 4-epi-tetracycline & $\mathrm{Fm}>\mathrm{T}$ & $<\mathrm{MRL}$ & 2.22 & $100 \%$ & $\mathrm{Fm}>\mathrm{T}$ & $<\mathrm{MRL}$ & 13.55 & $100 \%$ \\
\hline 4-epi-chlortetracycline & $\mathrm{Fm}>\mathrm{T}$ & $<\mathrm{MRL}$ & 1.05 & $100 \%$ & $\mathrm{Fm}>\mathrm{T}$ & $<\mathrm{MRL}$ & 3.90 & $100 \%$ \\
\hline
\end{tabular}


Table IV. Results of the validation for penicillin

\begin{tabular}{|c|c|c|c|c|c|c|c|c|c|c|c|c|}
\hline \multirow[b]{2}{*}{ Analyte } & \multicolumn{4}{|c|}{ Transition 1 « major» } & \multicolumn{4}{|c|}{ Transition 2 « minor » } & \multicolumn{4}{|c|}{ Transition 3 « minor » } \\
\hline & $\mathrm{Fm} / \mathrm{T} / \mathrm{B}$ & Ccbeta & $\mathrm{LOD}(\mu \mathrm{g} / \mathrm{kg})$ & Sensitivity (\%) & $\mathrm{Fm} / \mathrm{T} / \mathrm{B}$ & Ccbeta & LOD $(\mu \mathrm{g} / \mathrm{kg})$ & Sensitivity (\%) & $\mathrm{Fm} / \mathrm{T} / \mathrm{B}$ & Ccbeta & $\mathrm{LOD}(\mu \mathrm{g} / \mathrm{kg})$ & Sensitivity $(\%)$ \\
\hline Amoxicillin & $\mathrm{Fm}<\mathrm{B}$ & $>$ Cval & 1.74 & 81.7 & $\mathrm{~B}<\mathrm{Fm}<\mathrm{T}$ & $>$ Cval & 0.91 & 96.7 & $\mathrm{~B}<\mathrm{Fm}<\mathrm{T}$ & $>$ Cval & 1.18 & 95.00 \\
\hline Ampicillin & $\mathrm{Fm}>\mathrm{T}$ & $<$ Cval & 0.08 & 100.0 & $\mathrm{~B}<\mathrm{Fm}<\mathrm{T}$ & $>$ Cval & 0.97 & 98.3 & & & & \\
\hline Penicillin G & $\mathrm{Fm}>\mathrm{T}$ & $<$ Cval & 0.14 & 100.0 & $\mathrm{Fm}>\mathrm{T}$ & $<$ Cval & 0.17 & 100.0 & & & & \\
\hline Penicillin V & $\mathrm{Fm}>\mathrm{T}$ & $<$ Cval & 0.63 & 100.0 & $\mathrm{Fm}=\mathrm{T}$ & $<$ Cval & 1.39 & 98.3 & & & & \\
\hline Oxacillin & $\mathrm{Fm}>\mathrm{T}$ & $<$ Cval & 0.14 & 100.0 & $\mathrm{Fm}>\mathrm{T}$ & $<$ Cval & 0.24 & 100.0 & & & & \\
\hline Cloxacillin & $\mathrm{Fm}>\mathrm{T}$ & $<$ Cval & 2.08 & 100.0 & $\mathrm{Fm}>\mathrm{T}$ & $<$ Cval & 0.16 & 100.0 & & & & \\
\hline Nafcillin & $\mathrm{Fm}>\mathrm{T}$ & $<$ Cval & 0.06 & 100.0 & $\mathrm{Fm}>\mathrm{T}$ & $<$ Cval & 0.17 & 100.0 & & & & \\
\hline Dicloxacillin & $\mathrm{Fm}>\mathrm{T}$ & $<$ Cval & 0.43 & 100.0 & $\mathrm{Fm}>\mathrm{T}$ & $<$ Cval & 0.39 & 100.0 & & & & \\
\hline
\end{tabular}

